\title{
Appearance of Hagedorn limiting temperature in microscopic model calculations
}

\author{
E. Zabrodin ${ }^{1,2,3,4, a}$, L. Bravina ${ }^{1,3,4}$, M. Bleicher ${ }^{3,5}$, and H. Stöcker ${ }^{3,6,7}$ \\ ${ }^{1}$ Department of Physics, University of Oslo, PB 1048 Blindern, Oslo, Norway \\ ${ }^{2}$ Skobeltsyn Institute of Nuclear Physics, Moscow State University, RU-119991 Moscow, Russia \\ ${ }^{3}$ Frankfurt Institute for Advanced Studies, Ruth-Moufang-Str. 1, D-60438 Frankfurt a.M., Germany \\ ${ }^{4}$ National Research Nuclear University "MEPhl" (Moscow Engineering Physics Institute), RU-115409 \\ Moscow, Russia \\ ${ }^{5}$ Institute for Theoretical Physics, J.W. Goethe Universität, D-60438 Frankfurt a.M., Germany \\ ${ }^{6}$ J.W. Goethe Universität, D-60438 Frankfurt a.M., Germany \\ ${ }^{7}$ GSI Helmholtzzentrum für Schwerionenforschung GmbH, D-64291 Darmstadt, Germany
}

\begin{abstract}
One of important consequences of Hagedorn statistical bootstrap model is the prediction of limiting temperature $T_{\text {crit }}$ for hadron systems colloquially known as Hagedorn temperature. According to Hagedorn, this effect should be observed in hadron spectra obtained in infinite equilibrated nuclear matter rather than in relativistic heavy-ion collisions. We present results of microscopic model calculations for the infinite nuclear matter, simulated by a box with periodic boundary conditions. The limiting temperature indeed appears in the model calculations. Its origin is traced to strings and many-body decays of resonances.
\end{abstract}

\section{Introduction}

The statistical bootstrap model (SBM) is based on the assumption of infinite sequence of heavier and heavier resonances (or clusters), each being a constituent of a heavier resonance and, simultaneously, being composed of lighter ones. The bootstrap equation proposed by Hagedorn $[1,2]$ reads in a general form as

$$
\rho(m)_{m}=\{\text { input particles }\}+\sum_{2}^{\infty} \int \ldots \int\left\{\text { products of } \delta-\text { functions and } \rho^{\prime} s\right\},
$$

where $\rho(m)$ is a density of clusters with masses between $m$ and $m+d m$. This equation has an analytical solution [3]

$$
\rho(m)_{m \rightarrow \infty} \rightarrow \rho_{0} m^{a_{H}} \exp \left(m / T_{H}\right)
$$

containing two parameters: the exponent $a_{H} \approx-5 / 2$ and the limiting temperature $T_{H}$ above which the hadronic matter becomes unstable. This temperature is known nowadays simply as a Hagedorn temperature, and at $T \geq T_{H}$ the phase transition to a new state of matter, quark-gluon plasma (QGP),

\footnotetext{
a e-mail: eugen.zabrodin@fys.uio.no
} 
takes place. Further details of the SBM can be found elsewhere, see, e.g., [1, 2, 4-6] and references therein. Answering the question how the SBM is related to real collisions, Hagedorn admitted that [2] “... All this applies to infinitely extended hadronic matter in equilibrium. Experiments unfortunately produce only microscopic lumps of such matter, which are never in equilibrium. The relation between the described model and the situation in a collision is, therefore, far from trivial. It seems, however, that at any given time equilibrium is nearly reached locally, so that the model can be applied locally and then be folded with collective motions assumed ad hoc or derived from special models."

The microscopic transport models [7-10], employed for calculations of $h h, h A$ and $A+A$ collisions at relativistic energies, utilise the colored objects, i.e. strings, in addition to the tables of known hadrons and their resonances. Strings in their turn decay into hadrons, and the string masses are not limited from the top. Can this picture be similar to that proposed by the SBM? It is very tempting, therefore, to use the microscopic model with hadrons, resonances and strings for the simulation of infinite nuclear matter. Aspects of the calculations are discussed in Sec. 2.

\section{Simulation of infinite nuclear matter}

\section{A. Cubic box with periodic boundary conditions.}

The standard choice for such a simulation is a cubic box with periodic boundary conditions [11-13] to ensure the energy and momentum conservation. If one particle leaves the box, another particle, fully identical to the first one, enters the box from the opposite side. The box should be neither too big nor too small, e.g. $V=10 \times 10 \times 10=1000 \mathrm{fm}^{3}$ [11] or $V=5 \times 5 \times 5=125 \mathrm{fm}^{3}$ [12]. The energy density $\varepsilon$, the net baryon density $\rho_{B}$, and the net strangeness density $\rho_{S}$ are fixed at the initial stage. Recall, that for the infinite nuclear matter the net strangeness is zero, but for the central cell in relativistic heavy-ion collisions, which is an open system, $\rho_{S}$ can be small though differ from zero $[14,15]$. In case of $\rho_{S}=0$ the initial configuration in the box consists of protons and neutrons uniformly distributed in the configuration space. Their momenta are then rescaled to get the required energy density. The system is ready, and one has to employ a microscopic model, such as UrQMD [7, 8] or HSD [9], to trace the evolution of the system and study the relaxation of hadron-string matter in the box to equilibrium.

First, the kinetic equilibrium should be verified. It assumes that the velocity distributions $f_{i}\left(v_{j}\right), j=x, y, z$ of hadron species are close to Maxwellian and isotropic, $f_{i}\left(v_{x}\right)=f_{i}\left(v_{y}\right)=f_{i}\left(v_{z}\right)$, thus leading to isotropy of the pressure gradients. Then, the particle composition and their energy spectra should be time independent. This conditions correspond to requirements of chemical equilibrium and thermal equilibrium, respectively.

\section{B. Features of UrQMD model.}

The Ultra-relativistic Quantum Molecular Dynamics model (UrQMD) [7, 8] is a Monte-Carlo event generator designed for the description of hadron-hadron, hadron-nucleus, and nucleus-nucleus collisions in a broad energy range from hundred $\mathrm{MeV}$ up to several $\mathrm{TeV}$. As independent degrees of freedom the model contains 55 baryon and 32 meson states, together with their antiparticles and explicit isospin-projected states, with masses up to $2.25 \mathrm{GeV} / c$. At lower energies the interaction dynamics of $h h$ or $A+A$ collisions takes into account interactions between the hadrons and their excited states, resonances. At higher energies the UrQMD treats the production of new particles via formation and fragmentation of specific colored objects, strings. Strings are uniformly stretched between the quarks, diquarks and their antistates with constant string tension $\kappa \approx 1 \mathrm{GeV} / \mathrm{fm}$. The excited strings are fragmenting into pieces via the Schwinger mechanism of $q \bar{q}$-pair production, and the distribution of newly produced hadrons is uniform in the rapidity space.

UrQMD utilises the longitudinal mechanism of string excitation, where the string masses arise from momentum transfer. The model employs tables of the experimentally available information, 
such as hadron cross sections, resonance widths and decay modes. If this information is lacking, the one-boson exchange model, detailed balance considerations and isospin symmetry conditions are used. The propagation of particles is governed by Hamilton equation of motion, and hadronic cascade is used for the description of $h A$ and $A+A$ interactions. Due to the uncertainty principle newly produced particles can interact further only after a certain formation time. Hadrons containing the valence quarks, however, can interact immediately with the reduced cross section $\sigma=\sigma_{q N}$. The Pauli principle is taken into account via the blocking of the final state, if the outgoing phase space is occupied.

C. Statistical model of ideal hadron gas.

To extract the thermodynamic characteristics, such as temperature $T$, baryo-chemical potential $\mu_{B}$ and strangeness chemical potential $\mu_{S}$ one has to compare the hadron energy spectra and yields, extracted from the box calculations, with those obtained in statistical model (SM) of an ideal hadron gas [16-18] with essentially the same degrees of freedom. - If the system is in thermal and chemical equilibrium, its macroscopic characteristics are fully determined by particle distribution functions

$$
f\left(p, m_{i}\right)=\left[\exp \left(\frac{\sqrt{p^{2}+m_{i}^{2}}-\mu_{\mathrm{B}} B_{i}-\mu_{\mathrm{S}} S_{i}}{T}\right) \pm 1\right]^{-1},
$$

where $p, m_{i}$ and $d_{i}$ is the momentum and mass of the hadron species $i$, respectively. Sign + stands for fermions and - for bosons. One has to know just three parameters, namely, temperature $T$ and chemical potentials assigned to the conserved charges: baryon chemical potential $\mu_{\mathrm{B}}$ and strangeness chemical potential $\mu_{\mathrm{S}}$. Chemical potential of $i$-th hadron depends on baryon and strangeness content $\mu_{i}=B_{i} \mu_{\mathrm{B}}+S_{i} \mu_{\mathrm{S}}$. Then the expressions for particle number density $n_{i}$, energy density $\varepsilon_{i}$ and pressure $P$ read

$$
\begin{aligned}
n_{i} & =\frac{g_{i}}{2 \pi^{2}} \int_{0}^{\infty} p^{2} f\left(p, m_{i}\right) d p \\
\varepsilon_{i} & =\frac{g_{i}}{2 \pi^{2}} \int_{0}^{\infty} p^{2} \sqrt{p^{2}+m_{i}^{2}} f\left(p, m_{i}\right) d p \\
P & =\frac{g_{i}}{2 \pi^{2}} \int_{0}^{\infty} p^{2} d p \frac{p^{2}}{3\left(p^{2}+m_{i}^{2}\right)^{1 / 2}} f\left(p, m_{i}\right) .
\end{aligned}
$$

with $g_{i}$ being the spin-isospin degeneracy factor. The entropy density $s_{i}$ can be calculated either via the distribution function as

$$
s_{i}=-\frac{g_{i}}{2 \pi^{2}} \int f\left(p, m_{i}\right)\left[\ln f\left(p, m_{i}\right)-1\right] p^{2} d p,
$$

or from the Gibbs thermodynamic identity

$$
T s_{i}=\varepsilon_{i}+P_{i}-\mu_{B} \rho_{B_{i}}-\mu_{S} \rho_{S_{i}} .
$$

The procedure is as follows $[14,15]$. The values of energy density $\varepsilon$, baryon density $\rho_{B}$ and strangeness density $\rho_{S}$ employed for the box calculations are inserted as an input to a system of the SM nonlinear equations 


$$
\begin{aligned}
\varepsilon & =\sum_{i} \varepsilon_{i}^{\mathrm{SM}}\left(T, \mu_{\mathrm{B}}, \mu_{\mathrm{S}}\right), \\
\rho_{\mathrm{B}} & =\sum_{i} B_{i} \cdot n_{i}^{\mathrm{SM}}\left(T, \mu_{\mathrm{B}}, \mu_{\mathrm{S}}\right), \\
\rho_{\mathrm{S}} & =\sum_{i} S_{i} \cdot n_{i}^{\mathrm{SM}}\left(T, \mu_{\mathrm{B}}, \mu_{\mathrm{S}}\right) .
\end{aligned}
$$

to determine temperature $T$, baryon chemical potential $\mu_{B}$ and strangeness chemical potential $\mu_{S}$. After that all characteristics of the system in equilibrium are known and particle spectra can be compared with those obtained from microscopic model calculations.

\section{Relaxation to equilibrium}

Before starting the simulations one has to select energy density, net baryon density and net strangeness density in the system. As one of the possible benchmarks we choose the conditions corresponding to those in the central cell in central $\mathrm{Pb}+\mathrm{Pb}$ collisions at SPS energy $\left(E_{\text {lab }}=160 \mathrm{AGeV}\right)$ at $t=10 \mathrm{fm} / c$ after the beginning of the collision. At this moment $\varepsilon=468 \mathrm{GeV} / \mathrm{fm}^{3}, \rho_{B}=00.9 \mathrm{fm}^{-3}$ and $\rho_{S}=$ $-0.01 \mathrm{fm}^{-3}$, and the hadron matter in the cell is already in kinetic equilibrium [15]. Figure 1 displays the difference $\sigma_{x}-\left(\sigma_{y}+\sigma_{z}\right) / 2$ of the second moments $\sigma_{j}^{(2)}=\left\langle v_{j}^{2}\right\rangle-\left\langle v_{j}\right\rangle^{2}, j=x, y, z$ of the longitudinal and transverse velocity distributions of main hadron species.
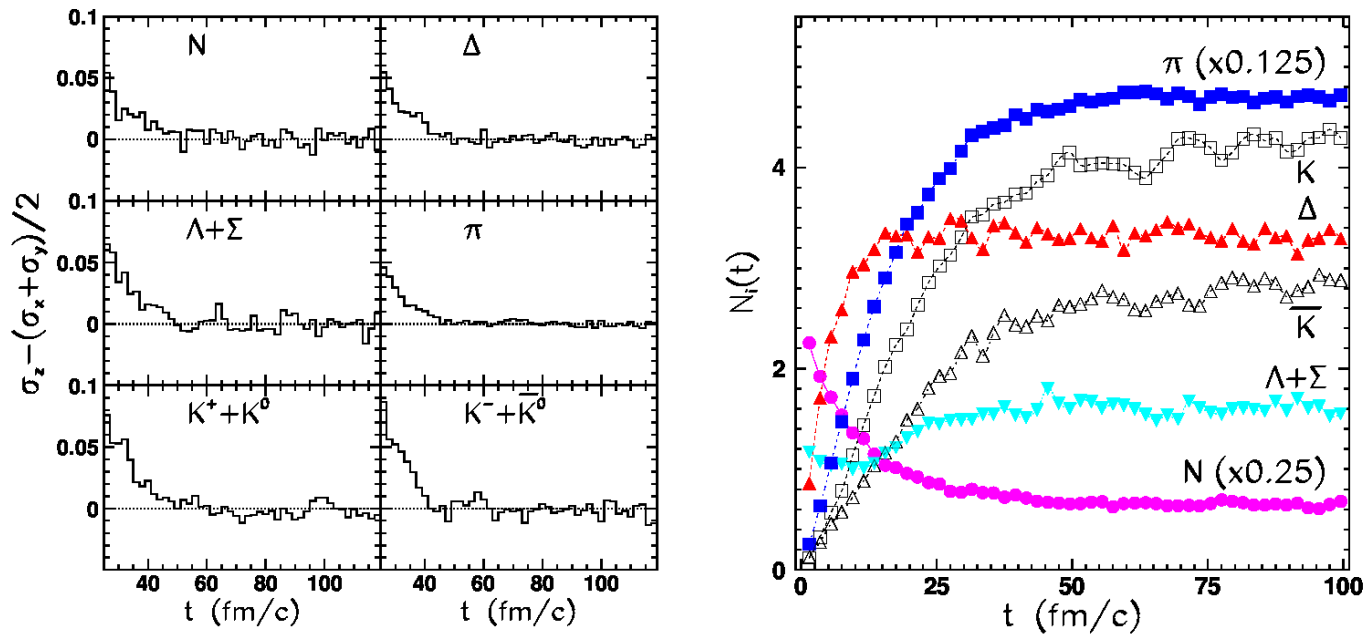

Figure 1. Anisotropy $\sigma_{x}-\left(\sigma_{y}+\sigma_{z}\right) / 2$ of the velocity Figure 2. Time evolution of the hadron yields in the distributions of hadrons in the box with $V=125 \mathrm{fm}^{3}$, box with the same parameters as those in Fig. 1. (From $\varepsilon=468 \mathrm{GeV} / \mathrm{fm}^{3}, \rho_{B}=00.9 \mathrm{fm}^{-3}$ and $\left.\rho_{S}=-0.01 \mathrm{fm}^{-3} \quad[12]\right)$.

as a function of time.

These distributions become isotropic at $t \approx 50 \mathrm{fm} / c$. Although the yields of some hadron species are also frozen at that time, as shown in Fig. 2, for kaons and antikaons the saturation takes place 
later, at $t \approx 90 \mathrm{fm} / c$ only. The late relaxation of kaons is not a feature of a particular model. Similar delays for the saturation times of strange particles were observed in [13] also. Recall, that although the chemical composition of hadrons in the box is frozen, the inelastic reactions can still take place [12].

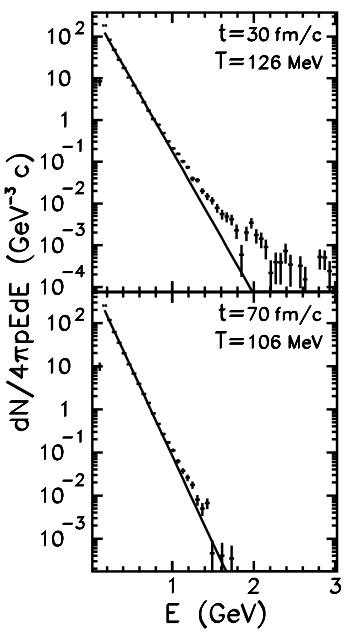

(a)

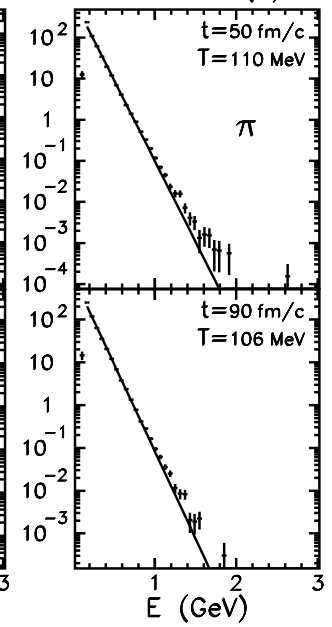

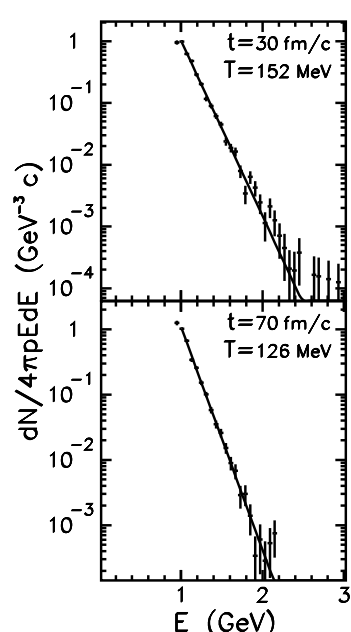

(b)

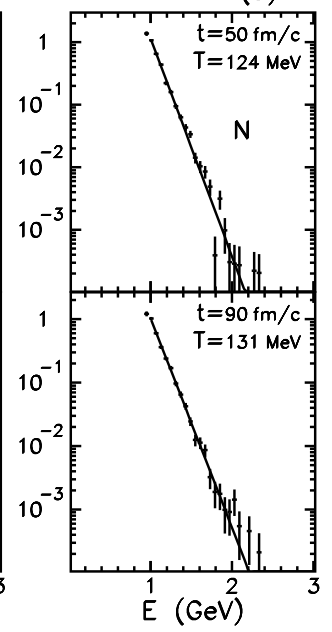

Figure 3. Energy spectra of (a) pions and (b) nucleons at 30, 50, 70 and $90 \mathrm{fm} / \mathrm{c}$ after the beginning of collisions in the box. Initial conditions and volume of the box are the same as in Fig. 1.

Finally, the conditions of thermal equilibrium should be investigated. The energy spectra of pions and nucleons at times from $30 \mathrm{fm} / c$ to $90 \mathrm{fm} / c$ are shown in Fig. 3(a) and Fig. 3(b), respectively. Lines indicate the results of the fit to Boltzmann-Gibbs distribution $\exp \left(-E_{i} / T+\mu_{i} / T\right)$. For pions the spectrum becomes time independent at $t \geq 70 \mathrm{fm} / c$ indicating that the thermal equilibrium is attained with the apparent temperature $T_{\pi} \approx 106 \mathrm{MeV}$. Nucleons seems to be thermalized already at $t \approx 50 \mathrm{fm} / c$ with a bit higher apparent temperature $T_{N} \approx 127 \mathrm{MeV}$. Still the difference between $T_{\pi}$ and $T_{N}$ is small, and the nuclear matter in the box reaches the state which is very close to thermal and chemical equilibrium.

One should not be confused here by the long times needed to reach an equilibrium state. The relaxation times to chemical and thermal equilibrium in the box, which is a closed system, are long because of the continuous production of new hadrons in energetic collisions of primary hadrons. For the realistic heavy-ion collisions equilibration process proceeds much faster. We will come to this point later in Sec. 5 .

\section{Appearance of limiting temperature}

To decide whether or not the hadron-resonance-string matter in the box reveals the limiting temperature, one has to repeat calculations similar to those in Sec. 3 with different energy densities. In UrQMD this was done in [11] for the box with $V=1000 \mathrm{fm}^{3}, \rho_{B}=0.16 \mathrm{fm}^{-3}$ and $\rho_{S}=0 \mathrm{fm}^{-3}$. Results of the calculations are depicted in Fig. 4. Although the maximum energy density shown in this figure is $1 \mathrm{GeV} / \mathrm{fm}^{3}$, the calculations have been performed up to $\varepsilon=5 \mathrm{GeV} / \mathrm{fm}^{3}$ [11]. The results 


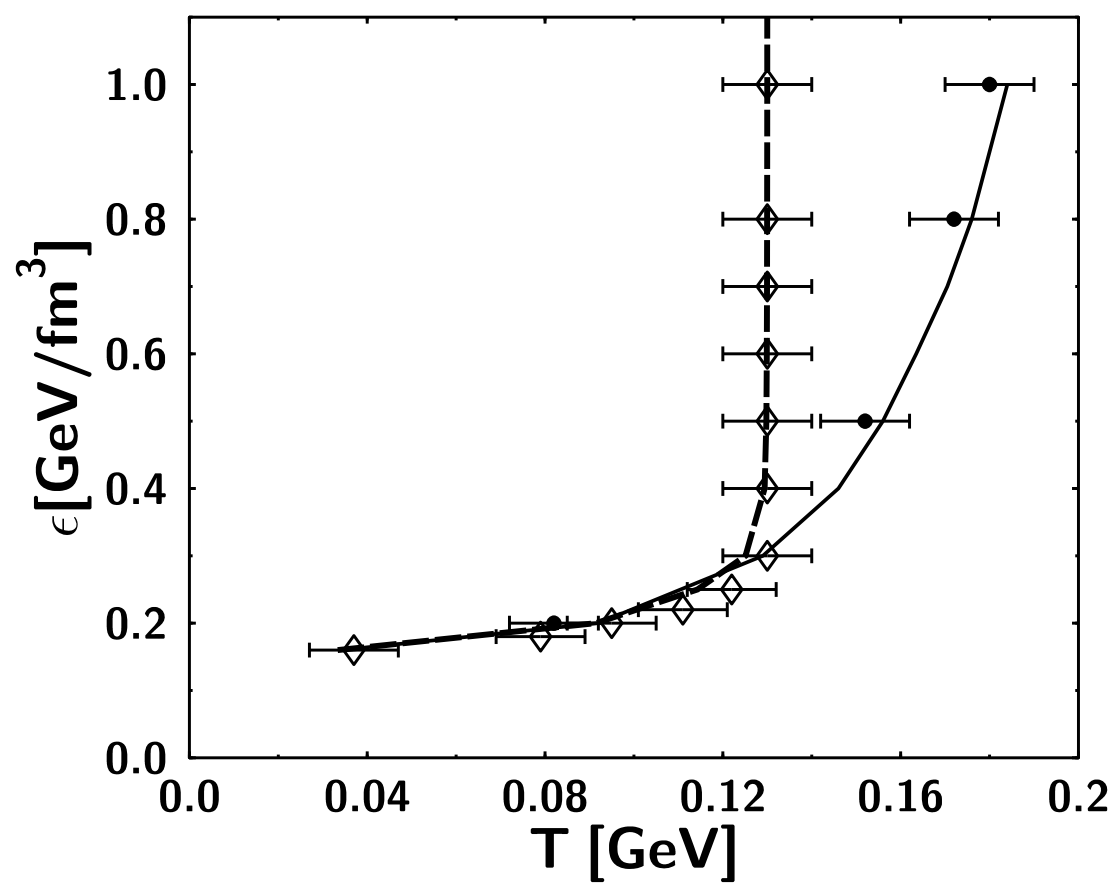

Figure 4. The energy density in the box with $V=1000 \mathrm{fm}^{3}$ versus the temperature. Open and full symbols show the UrQMD calculations with and without the strings and many-body resonance decays, respectively. Solid curve indicates the statistical model calculations, whereas dashed curve displays the SM calculations with the continuum mass spectrum. (From [11]).

remain stable, namely, at energy densities below $0.3 \mathrm{GeV} / \mathrm{fm}^{3}$ the temperature rapidly rises with increasing $\varepsilon$, whereas at higher energy densities the temperature of hadron species in the box saturates at around $T \approx 130 \pm 15 \mathrm{MeV}$ [11]. Solid curve in Fig. 4 represents the predictions of the statistical model of ideal hadron gas with all but strings degrees of freedom. We see that in the latter case the temperature continues to increase with rising energy density. The situation can be partially improved if the string degrees of freedom are omitted in the microscopic model. However, this is not the whole story. The SM trend with infinitely rising temperature can be fully reproduced in the UrQMD calculations only if strings and (this is important to stress) many-body $(n>2)$ decays of resonances are suppressed, as shown by the full points in Fig. 4. And vise versa, can one get the limiting temperature out of the statistical model calculations? The answer is "yes", provided the continuum mass spectrum is implemented. The Hagedorn mass spectrum for the strings given by Eq.(2) was inserted in the statistical model in [11]. Six types of strings were considered, i.e. baryon strings with different strangeness content $(B=1 ; S=0,-1,-2,-3)$, meson strings with $(B=0 ; S=0,1)$ and their antistates, respectively. The upper mass limit was fixed to $M_{\max }=1 \mathrm{TeV}$. In this case the system reveals the limiting temperature behavior, represented by the dashed curve in the same Figure.

Hadronic degrees of freedom and parameters of the string excitation play a crucial role for the evaluation of numerical value of $T_{\text {crit }}$. As seen in Fig. 5, in the microscopic model with shorter 


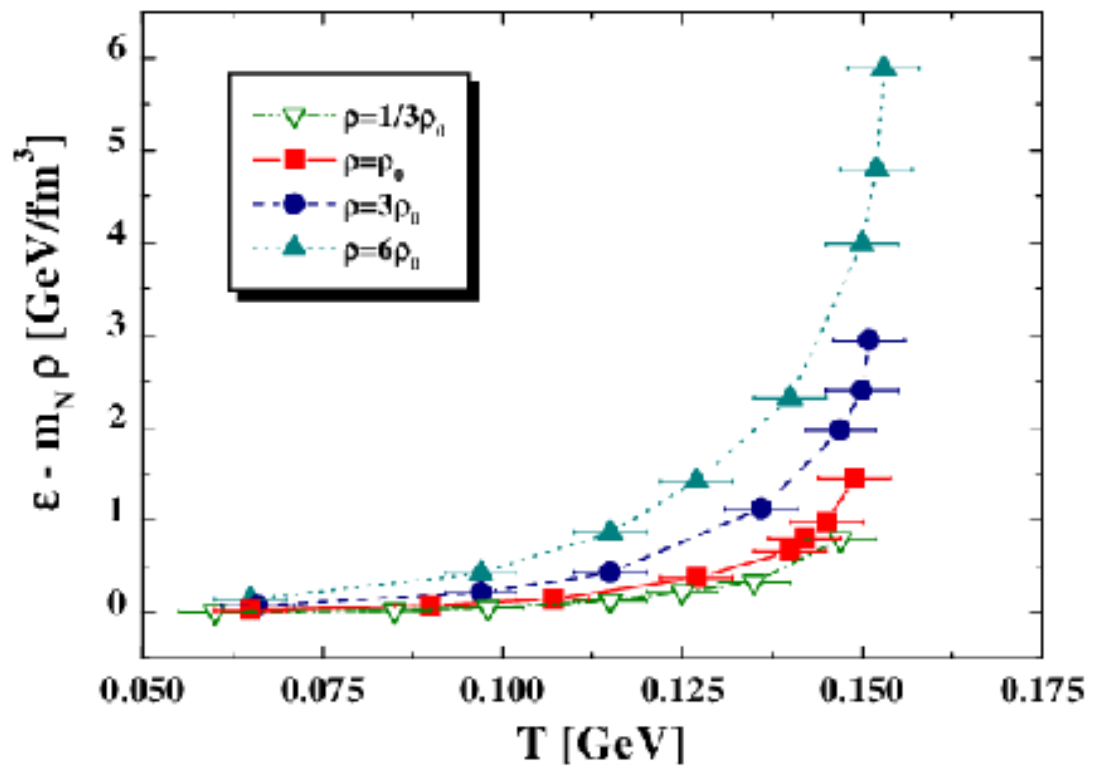

Figure 5. The same as Fig. 4 but for BUU+Lund transport model calculations [12]. The box volume is $V=$ $1000 \mathrm{fm}^{3}$, and the baryon density varies from $1 / 3 \rho_{0}$ to $6 \rho_{0}$. (From [13]).

table of resonances and different threshold for string excitations the limiting temperature is about $T_{\text {crit }} \approx 150 \pm 5 \mathrm{MeV}$ [13]. The $T_{\text {crit }}$, however, is remarkably insensitive to the baryon density of the system.

The abundances of all 55 baryon states and 32 meson states, implemented in UrQMD, are presented in Fig. 6 for the case of absence of strings and suppression of many-body decays of resonances. Predictions of the statistical model are plotted onto the UrQMD results also. The agreement between the two models is fair. Two meson species, $\eta^{\prime}$ and $f_{1}$, are missing in the model calculations simply because these mesons are produced in the UrQMD merely via the string fragmentation. This process was blocked in the calculations. Thus we may conclude that the presence of (i) strings and (ii) manybody decays of resonances in microscopic model calculations leads to emergence of Hagedorn-like limiting temperature in particle spectra.

Last but not least, we should check another aforementioned Hagedorn statement about the possibility of local equilibrium in relativistic heavy-ion collisions and compare the results with those obtained for the infinite matter. This comparison is done in Sec. 5. 

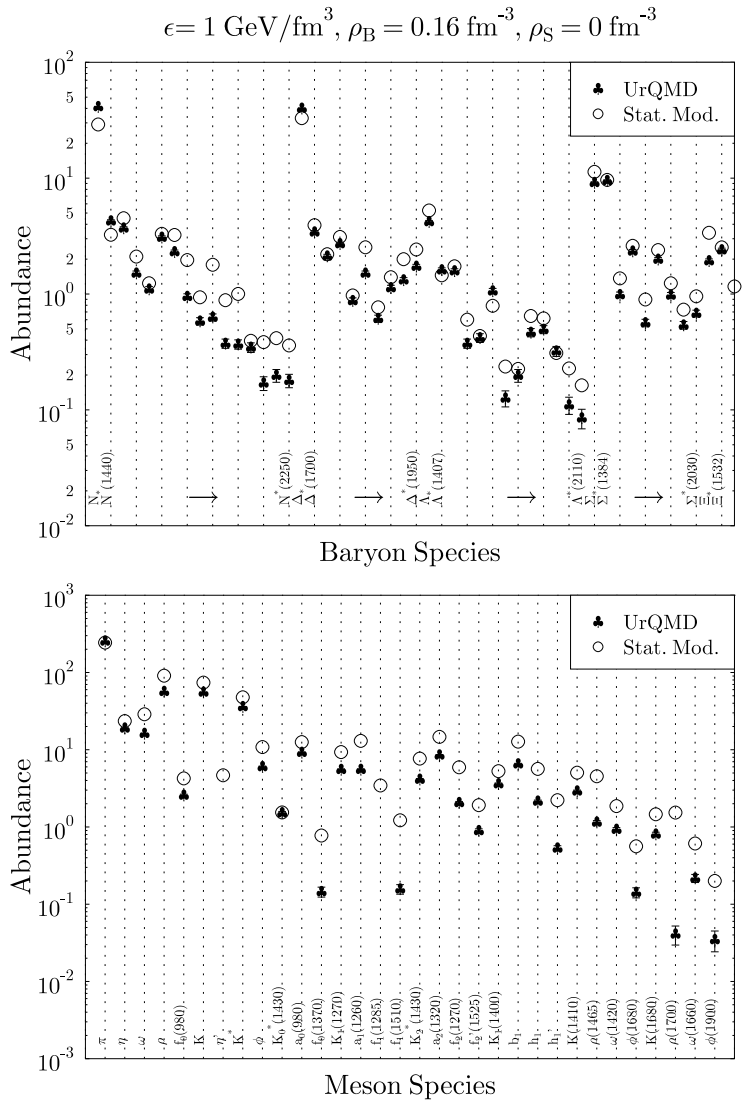

Figure 6. Yields of baryons (upper plot) and mesons (bottom plot) in UrQMD box calculations (solid symbols) and in statistical model of an ideal hadron gas (open symbols). Parameters of the box are $V=1000 \mathrm{fm}^{3}$, $\rho_{B}=0.16 \mathrm{fm}^{-3}, \rho_{S}=0 \mathrm{fm}^{-3}$. Strings and many-body decays of resonances are suppressed in the UrQMD calculations. (From [11]).

\section{Equilibrium in the central cell and in the box}

Two reactions will be discussed here, namely, central collisions of gold nuclei at AGS $\left(E_{l a b}=\right.$ $10.7 \mathrm{AGeV})$ and lead nuclei at SPS $\left(E_{l a b}=160 \mathrm{AGeV}\right)$. Results for other energies, such as $E_{l a b}=40 \mathrm{AGeV}$ or $\sqrt{s_{N N}}=200 \mathrm{GeV}$ can be found in $[19,20]$. Energy spectra of main hadron species in both reactions are displayed in Fig. 7. Here results obtained for the central cell of volume $V=125 \mathrm{fm}^{3}$ at $t=10 \mathrm{fm} / c$ after the beginning of the collision are compared with those extracted from the box calculations with the essentially the same values of $\varepsilon, \rho_{B}, \rho_{S}$. The agreement between the cell and the box results is good for both energies. - Note, however, that the deviations from the $\mathrm{SM}$ of an ideal hadron gas become stronger with rising collision energy. - Why the hadronic matter in the cell reaches the (quasi)equilibrium much earlier compared to the matter in the box? The cell is an 

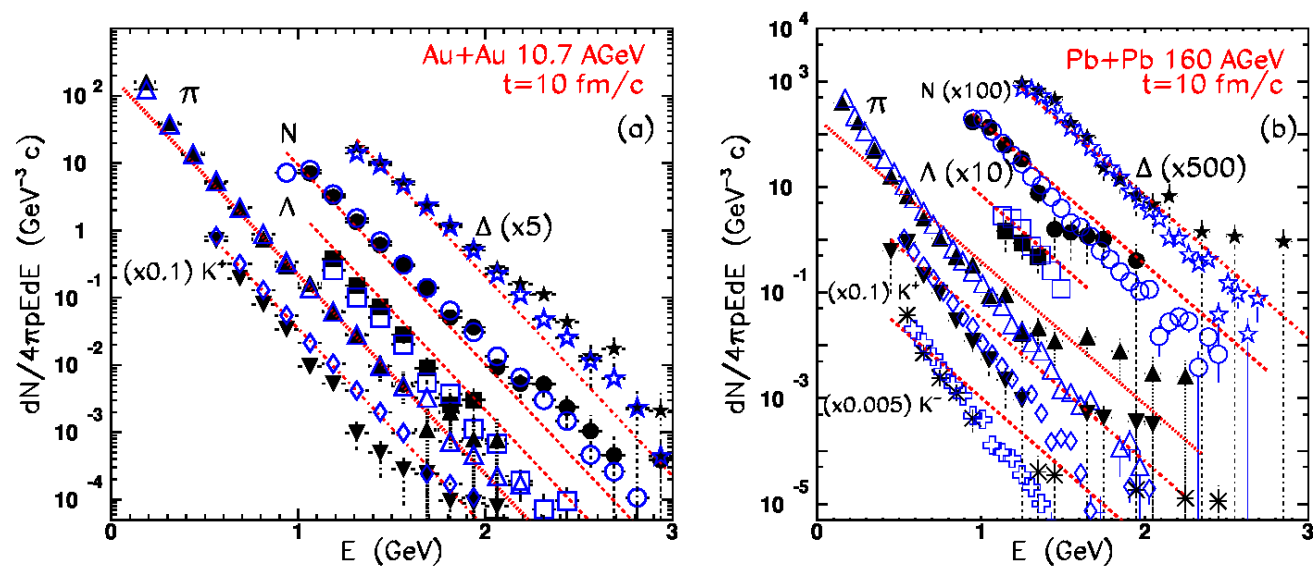

Figure 7. (a) Solid symbols: energy spectra of hadrons in the UrQMD calculations of central cell with $V=$ $125 \mathrm{fm}^{3}$ at $t=10 \mathrm{fm} / c$ of Au+Au collisions at $E_{\text {lab }}=10.7 \mathrm{AGeV}$. Open symbols denote the UrQMD box calculations with the same $\varepsilon, \rho_{B}, \rho_{S}$ as in the cell. Lines are results of the fit to the SM with the parameters $T=$ $147 \mathrm{MeV}, \mu_{B}=510 \mathrm{MeV}$ and $\mu_{S}=129 \mathrm{MeV}$. (b) The same as (a) but for $\mathrm{Pb}+\mathrm{Pb}$ collisions at $E_{\text {lab }}=160 \mathrm{AGeV}$.

open system, and energetic particles leave it quickly, thus resulting in effective "cooling" and faster thermalization of the rest matter in the cell compared to the box, which is a closed system where the total energy must be redistributed among all newly produced hadrons.

Still, the particle composition in the cell should be examined. We present the time evolution of hadron abundances in the cell in both reactions in Figs. 8(a) and 8(b). Here the values of $\varepsilon, \rho_{B}, \rho_{S}$ were extracted from the cell at each moment $t$ and used as an input for the box calculations, also plotted in these figures. Hadron multiplicities in the cell and in the box are close to each other at $t \geq 10 \mathrm{fm} / c$. It is interesting to note that the the fraction of already "frozen" particles in the cell is quite small compared to the total abundances. Statistical model provides a good agreement for all hadrons except pions, which are underestimated. The difference becomes stronger with rising collision energy and can be attributed to lack of detailed balance in microscopic models.

Nevertheless, equilibration in the central cell sets in quite late at $t \approx 10 \mathrm{fm} / c$ compared to $\tau_{0} \approx 0.5 \div 1 \mathrm{fm} / c$ used in hydrodynamic models for relativistic heavy-ion collisions. Recall, that many distributions in the cell, e.g. $P$ vs. $\varepsilon$ or entropy-per-baryon ratio $[15,19]$, indicate that quasiequilibrium description can be applicable much earlier, already at $t \approx 3-4 \mathrm{fm} / c$. These results are supported by the conclusions of Ref.[21], where the authors demonstrate the applicability of hydrodynamic description (under a certain conditions) to the systems being out of local equilibrium.

Thermalization rates can also be significantly increased if one directly includes the Hagedorn states, or resonances, with an exponential mass spectrum [22-24] in a hadron-resonance gas model. The simultaneous collision of several particles, most likely pions, cannot create a string, whereas it can produce a massive resonance called Hagedorn state (HS) [22]. This HS is a highly unstable state with a large decay width, therefore, the process of thermalization is speeded up extremely via the 
(a)

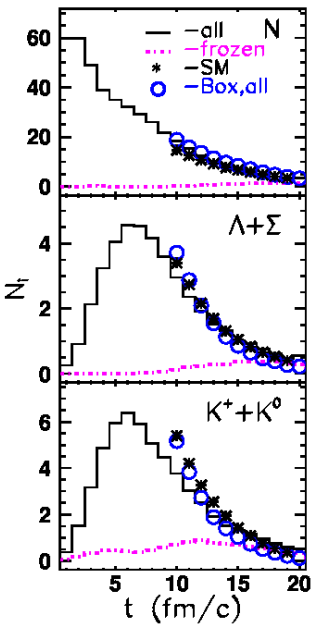

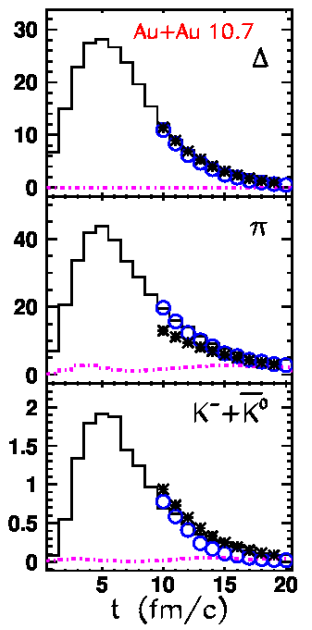

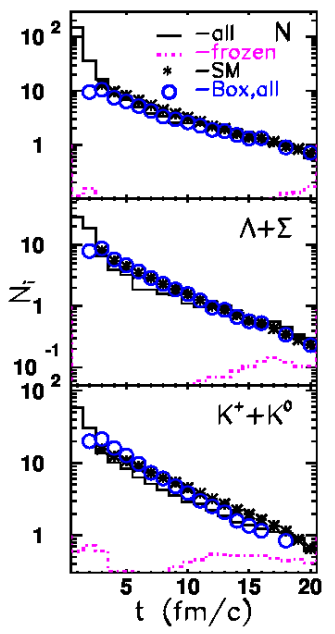

(b)

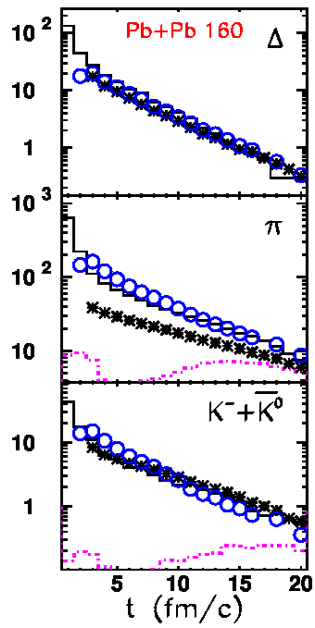

Figure 8. (a) The hadron yields in the central cell of Au+Au central collision at AGS energy (histograms) in comparison with the box calculations (open symbols) and predictions of SM (asterisks). Dash-dotted histograms show the fractions of already frozen particles in the cell. (b) The same as (a) but for $\mathrm{Pb}+\mathrm{Pb}$ central collisions at SPS energy. (From [12]).

reactions like

$$
n \pi \rightarrow\{H S\} \rightarrow n^{\prime} \pi+\{\bar{K} K+\bar{B} B+\ldots\}
$$

For instance, the times to reach chemical equilibrium for kaons and lambdas in the UrQMD box calculations are shortened to $2 \mathrm{fm} / c$ and $5 \mathrm{fm} / c$, respectively [24]. Proper inclusion of the Hagedorn states to microscopic transport models deserves further investigations.

\section{Conclusions}

In conclusion, relaxation of hot and dense hadron-string matter to chemical and thermal equilibrium is studied. Two cases are considered. The first one is the infinite nuclear matter (closed system), represented by a box with periodic boundary conditions. The second one is a central cell (open system) in central heavy-ion collisions at ultrarelativistic energies. In the box calculations the hadron abundances saturate after a certain time, which is longer for strange particles compared to the non-strange ones. Energy spectra of different hadrons exhibit two time-independent inverse slopes corresponding to two temperatures, $T_{\text {meson }}$ and $T_{\text {baryon }}$. With the rise of the energy density in the system these spectra show the existence of the Hagedorn-like limiting temperature $T_{c r i t}=130 \pm 15 \mathrm{MeV}$ (UrQMD) or $T_{c r i t}=150 \pm 5 \mathrm{MeV}$ (BUU+Lund). The absolute value of the $T_{\text {crit }}$ depends on the table of resonances in the particular model and on the parameters of the string excitation and fragmentation. The origin of limiting temperature in the model is traced to presence of strings and many-body decays of resonances. Comparison with the statistical model of an ideal hadron gas shows that the SM is able to reproduce the UrQMD box limiting temperature behavior, provided it utilises the continuum Hagedorn-like mass spectrum of strings in addition to hadrons and resonances. 
The yields and energy spectra of hadron species in the cell at $t \geq 10 \mathrm{fm} / c$ are very close to those in the box with the same values of energy density, net baryon density, and net strangeness density. The obtained results are independent on the collision energy of nuclei. The relaxation time can be significantly reduced if one allows for creation of Hagedorn states with exponential mass spectrum in the vicinity of $T_{\text {crit }}$.

\section{References}

[1] R. Hagedorn, Nuovo Cim. Suppl. 3, 147 (1965)

[2] R. Hagedorn, Riv. Nuovo Cim. 6N10, 1 (1983)

[3] W. Nahm, Nucl. Phys. B45, 525 (1972)

[4] M. Gorenstein, these Proceedings

[5] K. Redlich, these Proceedings

[6] J. Rafelski (Ed.), Melting Hadrons, Boiling Quarks - From Hagedorn Temperature to UltraRelativistic Heavy-Ion Collisions at CERN (Springer, Heidelberg, 2016)

[7] S.A. Bass et al., Prog. Part. Nucl. Phys. 41, 255 (1998)

[8] M. Bleicher et al., J. Phys. G25, 1859 (1999)

[9] W. Ehehalt and W. Cassing, Nucl. Phys. A602, 449 (1996)

[10] N.S. Amelin, L.V. Bravina, Sov. J. Nucl. Phys. 51 (1990) 133;

L.V. Bravina et al., Phys. Lett. B354, 196 (1995)

[11] M. Belkacem et al., Phys. Rev. C58, 1727 (1998)

[12] L.V. Bravina et al., Phys. Rev. C62, 064906 (2000)

[13] E.L. Bratkovskaya et al., Nucl. Phys. A675, 661 (2000)

[14] L.V. Bravina et al., Phys. Lett. B434, 379 (1998);

L.V. Bravina et al., J. Phys. G25, 351 (1999)

[15] L.V. Bravina et al., Phys. Rev. C60, 024904 (1999);

L.V. Bravina et al., Nucl. Phys. A661, 600 (1999)

[16] J. Cleymans, K. Redlich, Phys. Rev. C60, 054908 (1999)

[17] A. Andronic, P. Braun-Munzinger, J. Stachel, Nucl. Phys. A772, 167 (2006).

[18] G. Torrieri et al., Comput. Phys. Commun. 167, 229 (2005)

[19] L.V. Bravina et al., Phys. Rev. C78, 014907 (2008);

E.E. Zabrodin et al., J. Phys. G36, 064065 (2009)

[20] L.V. Bravina et al., Phys. Rev. C63, 064902 (2001);

L.V. Bravina et al., Nucl. Phys. A698, 383c (2002);

L.V. Bravina et al., J. Phys. G27, 421 (2001)

[21] Ph. Mota, T. Kodama, R.D. de Souza, J. Takahashi, Eur. Phys. J. A48, 165 (2012)

[22] C. Greiner, S. Leupold, J. Phys. G27, L95 (2001)

[23] J. Noronha-Hostler, C. Greiner, I. Shovkovy, Phys. Rev. Lett. 100, 252301 (2008)

[24] M. Beitel, K. Gallmeister, C. Greiner, Phys. Rev. C90, 045203 (2014) 